\title{
Decay of massive scalar field in a Schwarzschild background
}

\author{
R.A. Konoplya \\ Universidade de São Paulo, Instituto de Física \\ Caixa Postal 66318, 05315-970, São Paulo-SP, Brazil. \\ E-mail: konoplya_roma@yahoo.com. \\ and \\ A.V. Zhidenko \\ Department of Physics, Dniepropetrovsk National University \\ St. Naukova 13, Dniepropetrovsk 49050, Ukraine. \\ E-mail: Z_A_V@ukr.net.
}

\begin{abstract}
The decay of massive scalar field in the Schwarzschild black hole background is investigated here by consideration its quasinormal spectrum. It has been proved that the so-called quasi - resonant modes, which are arbitrary long living (purely real) modes, can exist only if the effective potential is not zero at least at one of the boundaries of the $R$-region. We have observed that the quasinormal spectrum exists for all field masses and proved both analytically and numerically that when $n \rightarrow \infty$ the real part of the frequencies approaches the same asymptotical value $(\ln 3 /(8 \pi M))$ as in the case of the massless field.
\end{abstract}

At late times, the decay of fields in a black hole background is dominated by some resonant quasinormal modes (QNMs) [1. The interest in QNMs originated from possibility of detection of gravitational waves from colliding black holes, especially after creating the new generation of gravitational antennas. Recently the study of the quasinormal modes has gained a strong motivation coming from ADS/CFT correspondence, where the QNMs can be interpreted as the poles of the temperature Green function in the dual conformal field theory. It is also possible, that QNMs play an important role in Loop Quantum Gravity. All this motivated the extensive research of QNMs for different black holes and different fields (both massive and massless) around them (see for instance [2] and references therein).

Long time ago, when considering the problem of a massive scalar field around Schwarzschild black hole, A. Starobinskii and I. Novikov found, that, when working in the in the frequency domain, the poles of the Green function in the complex plane are closer to the real axis than in the massless case. Thus, it was observed that the "massive" modes decay more slowly than the massless ones. Later it was confirmed in a series of papers, both in frequency domain [3], 4], and in time domain [5]- [8, that the greater the mass of the field, the slower the decay. Then, a natural question is, what will happen at further increasing of the mass of the field? Can the decreasing decay rate approach zero leading to existence of arbitrary long living modes? Which is high overtone behavior in this case?

In the present paper we are answering these questions by thorough investigation of the quasinormal behavior corresponding to the decay of a massive scalar field.

1. Basic equations

The massive scalar field in a curved background is governed by the Klein-Gordon equation:

$$
\square \Phi-m^{2} \Phi=\frac{1}{\sqrt{-g}}\left(g^{\mu \nu} \sqrt{-g} \Phi, \mu\right){ }_{\nu}-m^{2} \Phi=0 .
$$

After the separation of angular and time variables, the result radial equation for the Schwarzschild background

$$
d s^{2}=f(r) d t^{2}-\frac{d r^{2}}{f(r)}-r^{2}\left(d \theta^{2}-\sin ^{2} \theta d \phi^{2}\right), \quad f(r)=1-\frac{2 M}{r},
$$

can be reduced to the following wave-like equation

$$
\left(\frac{d^{2}}{d r^{* 2}}+\omega^{2}-V\left(r^{*}\right)\right) \Psi\left(r^{*}\right)=0
$$


where

$$
d r^{*}=\frac{d r}{f(r)}, \quad V(r)=f(r)\left(\frac{l(l+1)}{r^{2}}+\frac{f^{\prime}(r)}{r}+m^{2}\right),
$$

and $l=0,1,2,3 \ldots$ parameterizes the field angular momentum.

The effective potential has the form of the potential barrier which approaches some constant values both at the event horizon and at spatial infinity. Therefore the QNMs boundary conditions (within the dominant asymptotic order) have the form:

$$
\Psi\left(r^{*}\right) \sim C_{ \pm} \exp \left( \pm i \kappa_{ \pm} r^{*}\right), \quad r^{*} \rightarrow \pm \infty ;
$$

Under these boundary conditions the QNMs of massive scalar field were studied in several papers in the WKB approximations [3, 4, 9]. Yet, if we need to explore accurate Leaver method [13], we have to deal with irregular singular point at infinity. This implies that one needs to take into consideration the sub-dominant asymptotic term at infinity:

$$
\Psi\left(r^{*}\right) \sim C_{+} e^{i \chi r^{*}} r^{\left(i M m^{2} / \chi\right)}, \quad\left(r, r^{*} \rightarrow+\infty\right) ; \quad \chi=\sqrt{\omega^{2}-m^{2}} .
$$

(The sign of $\chi$ is to be chosen to stay in the same complex surface quadrant as $\omega$.)

Within the Leaver method we can eliminate the singular factor of the solution (3) that satisfies ingoing wave boundary condition at the horizon and (5) at infinity, and expand the remaining part into the Frobenius series that are convergent in the $R$-region (between the event horizon and the infinity). The appropriate series are:

$$
\Psi(r)=e^{i \chi r} r^{\left(2 i M \chi+i M m^{2} / \chi\right)}\left(1-\frac{2 M}{r}\right)^{-2 i M \omega} \sum_{n} a_{n}\left(1-\frac{2 M}{r}\right)^{n},
$$

Substituting (6) into (3) we obtain a three-term recurrent relation for the coefficients $a_{n}$ :

$$
\begin{aligned}
& \alpha_{0} a_{1}+\beta_{0} a_{0}=0 ; \\
& \alpha_{n} a_{n+1}+\beta_{n} a_{n}+\gamma_{n} a_{n-1}=0, \quad n>0,
\end{aligned}
$$

where

$$
\begin{aligned}
\alpha_{n} & =(n+1)(n+1-4 M i \omega) ; \\
\beta_{n} & =\frac{M(\omega+\chi)\left(4 M(\omega+\chi)^{2}+i(2 n+1)(\omega+3 \chi)\right)}{\chi}-2 n(n+1)-1-l(l+1) ; \\
\gamma_{n} & =\left(n-M i(\omega+\chi)^{2} / \chi\right)^{2} .
\end{aligned}
$$

Since the series are convergent at infinity, the ratio of the series coefficients is finite and can be found in two ways:

$$
\frac{a_{n+1}}{a_{n}}=\frac{\gamma_{n}}{\alpha_{n}} \frac{\alpha_{n-1}}{\beta_{n-1}-\frac{\alpha_{n-2} \gamma_{n-1}}{\beta_{n-2}-\alpha_{n-3} \gamma_{n-2} / \ldots}}-\frac{\beta_{n}}{\alpha_{n}}=-\frac{\gamma_{n+1}}{\beta_{n+1}-\frac{\alpha_{n+1} \gamma_{n+2}}{\beta_{n+2}-\alpha_{n+2} \gamma_{n+3} / \ldots}} .
$$

Thus we have equation with respect to the eigenvalue $\omega$ :

$$
\beta_{n}-\frac{\alpha_{n-1} \gamma_{n}}{\beta_{n-1}-\frac{\alpha_{n-2} \gamma_{n-1}}{\beta_{n-2}-\alpha_{n-3} \gamma_{n-2} / \ldots}}=\frac{\alpha_{n} \gamma_{n+1}}{\beta_{n+1}-\frac{\alpha_{n+1} \gamma_{n+2}}{\beta_{n+2}-\alpha_{n+2} \gamma_{n+3} / \ldots}},
$$

that can be solved numerically.

To improve the convergence of the continued fraction on the right side of (10) we used the technique developed by Nollert 14. He considered the recurrence relation

$$
R_{N}=\frac{\gamma_{N}}{\beta_{N}-\frac{\alpha_{N} \gamma_{N+1}}{\beta_{N+1}-\alpha_{N+1} \gamma_{N+2} / \ldots}}=\frac{\gamma_{N}}{\beta_{N}-\alpha_{N} R_{N+1}} .
$$


Making use of the recurrence relation (11) one can find for large N:

$$
R_{N}=C_{0}+C_{1} N^{-1 / 2}+C_{2} N^{-1}+\ldots
$$

where

$$
\begin{gathered}
C_{0}=-1, \\
C_{1}= \pm 2 \sqrt{-i M \chi}, \quad \operatorname{Re}\left(C_{1}\right)>0, \\
C_{2}=\frac{3}{4}+4 M i \chi+i M m^{2} / \chi,
\end{gathered}
$$

etc.

The series (12) converge for $|\chi| / N<A<\infty$, therefore we can use this approximation for $R_{N}$ inside the continued fraction for some $N \gg-\operatorname{Im}(\chi) \sim n$. In practice, to find an appropriate $N$ we should increase it until the result of the continued fraction calculation does not change.

2.Quasi-resonances

Recently Ohashi and Sakagami 12 studied QNMs for the decay of the massive scalar field and found that there are perturbations with arbitrary long life when the field mass has special values. They called these modes quasi-resonances.

The remarkable fact is that in the massive case the purely real frequencies are not forbidden. Indeed, by multiplying (3) by $\Psi^{*}$ and integrating we obtain:

$$
\int_{-R_{1}}^{+R_{2}} \Psi^{*}\left(\Psi^{\prime \prime}+\left(\omega^{2}-V\right) \Psi\right) d r^{*}=\omega^{2} \int_{-R_{1}}^{+R_{2}}|\Psi|^{2} d r^{*}-\int_{-R_{1}}^{+R_{2}}\left(\left|\Psi^{\prime}\right|^{2}+V|\Psi|^{2}\right) d r^{*}+\left.\Psi^{*} \Psi^{\prime}\right|_{r^{*}=-R_{1}} ^{r^{*}=+R_{2}}=0 .
$$

Substituting the boundary conditions for large $R_{1,2}$, we find that

$$
\begin{array}{r}
\left.\Psi^{*} \Psi^{\prime}\right|_{r^{*}=-R_{1}} ^{r^{*}=+R_{2}}=i \exp \left(-2 \operatorname{Im}(\chi) R_{2}\right) R_{2}^{2 M \operatorname{Im}(\chi) m^{2} /|\chi|^{2}}\left(\chi\left|C_{+}\right|^{2}+o\left(\frac{1}{R_{2}}\right) R_{2}\right)+ \\
+i \exp \left(-2 \operatorname{Im}(\omega) R_{1}\right)\left(\omega\left|C_{-}\right|^{2}+o\left(\frac{1}{R_{1}}\right) R_{1}\right) .
\end{array}
$$

It is easy to see that for the massless case $(\chi=\omega)$ and $\operatorname{Im}(\omega)=0$, the equation (13) implies

$$
\omega\left|C_{+}\right|^{2}=0 \quad \text { and } \quad \omega\left|C_{-}\right|^{2}=0 .
$$

This means that there is no real frequency in the QNM spectrum of the massless scalar field.

When the field mass is not zero, the purely real frequencies $\omega$ are not forbidden because (13) is satisfied when

$$
\operatorname{Re}(\chi)\left|C_{+}\right|^{2}=0, \quad \text { and } \quad \omega\left|C_{-}\right|^{2}=0 .
$$

There is no wave falling on the horizon $\left(C_{-}=0\right)$ in the latter case, and there is no energy transmission to infinity $(\operatorname{Re}(\chi)=0)$. Therefore oscillations do not decay. The situation is similar to the standing waves on a fixed string. The requirement for $\chi$ to be imaginary, bounds the quasi-resonance frequencies by the field mass

$$
\left|\omega_{Q R M}\right|<m \text {. }
$$

Eq. (15) also illustrates that phenomenon of quasi-resonance exists since the potential is not zero at spatial infinity. If the potential is zero for $r^{*} \rightarrow \pm \infty$ no quasi-resonant oscillations can exist. Thus, for instance, in the spectrum of the Schwarzschild-de Sitter background [17, no quasi-resonant oscillations can exist even for massive scalar field.

The numerical investigation shows, that increasing of the field mass gives rise to decreasing of the imaginary part of the QNM until reaching the vanishing damping rate. When some threshold values of $m$ are exceeded, the particular QN modes disappear (see figures 10 and 2). We can see, that the larger field mass is, the more first overtones share this destiny. Thus disappearing of the QNM does not induce the disappearing of the whole QN spectrum. Since, as it was proved in [16], the number of QN 


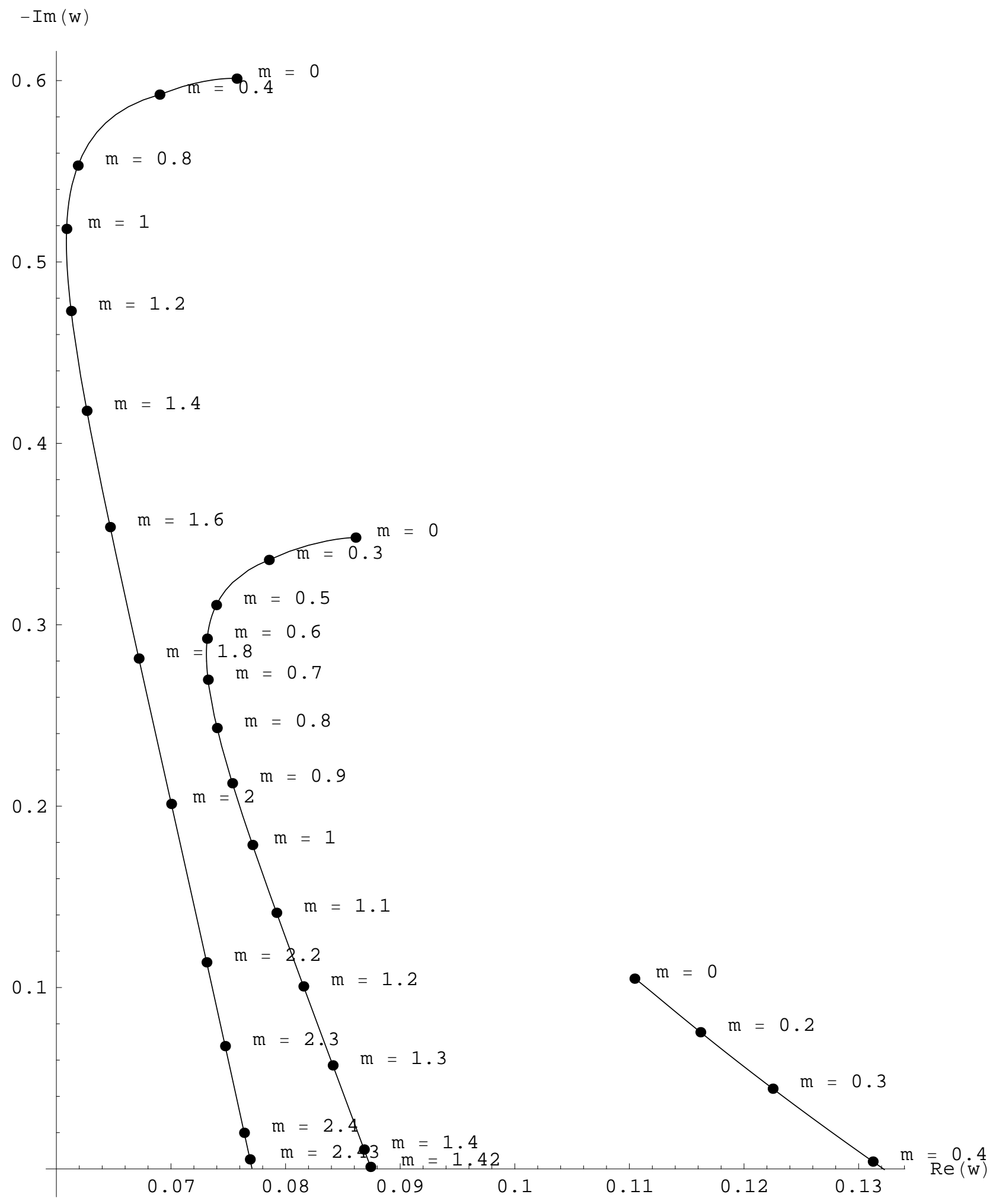

Figure 1: First three overtones of $l=0$ QNMs of the scalar field of different field masses $(\mathrm{M}=1)$ 


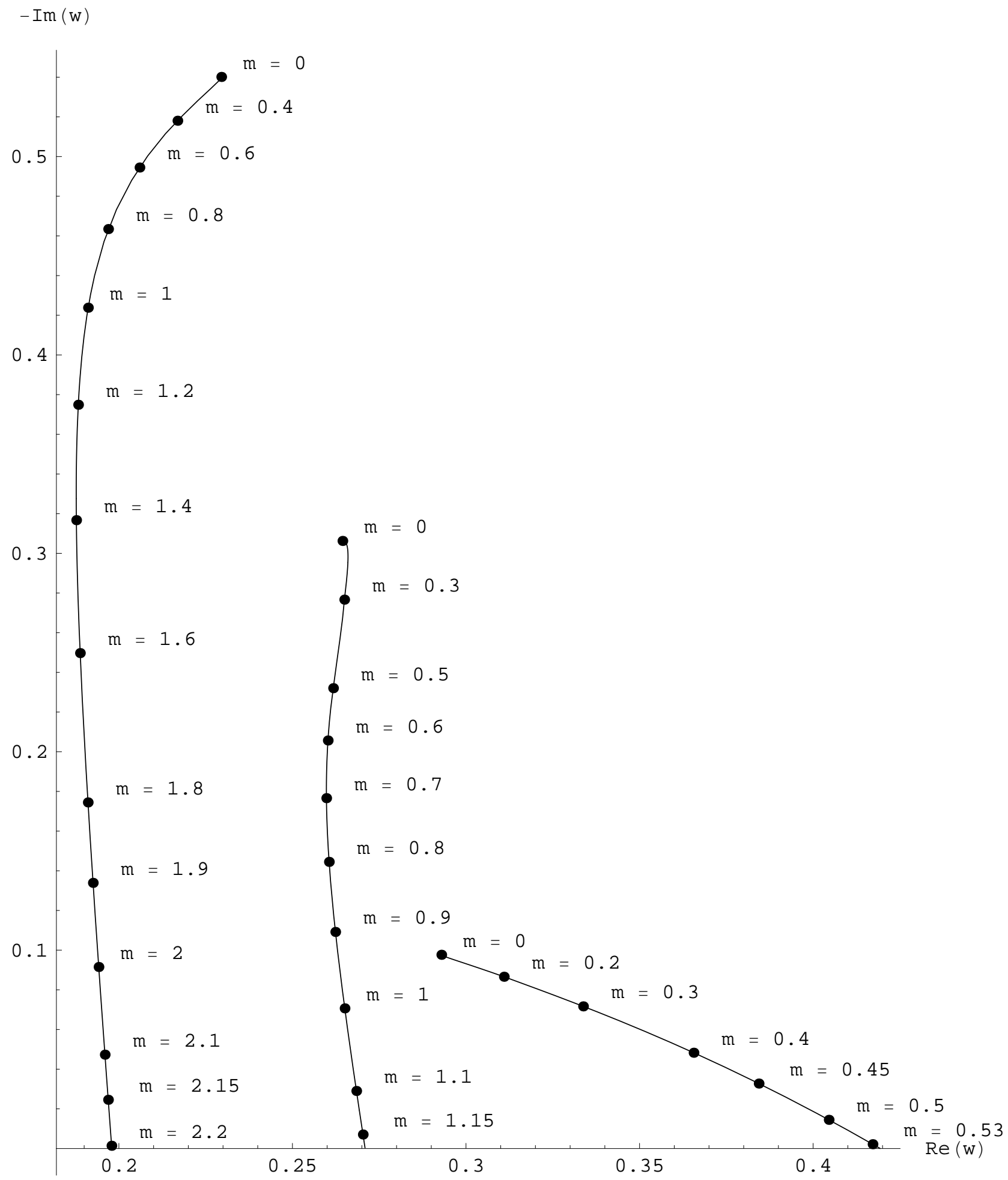

Figure 2: First three overtones of $l=1$ QNMs of the scalar field of different field masses $(\mathrm{M}=1)$ 


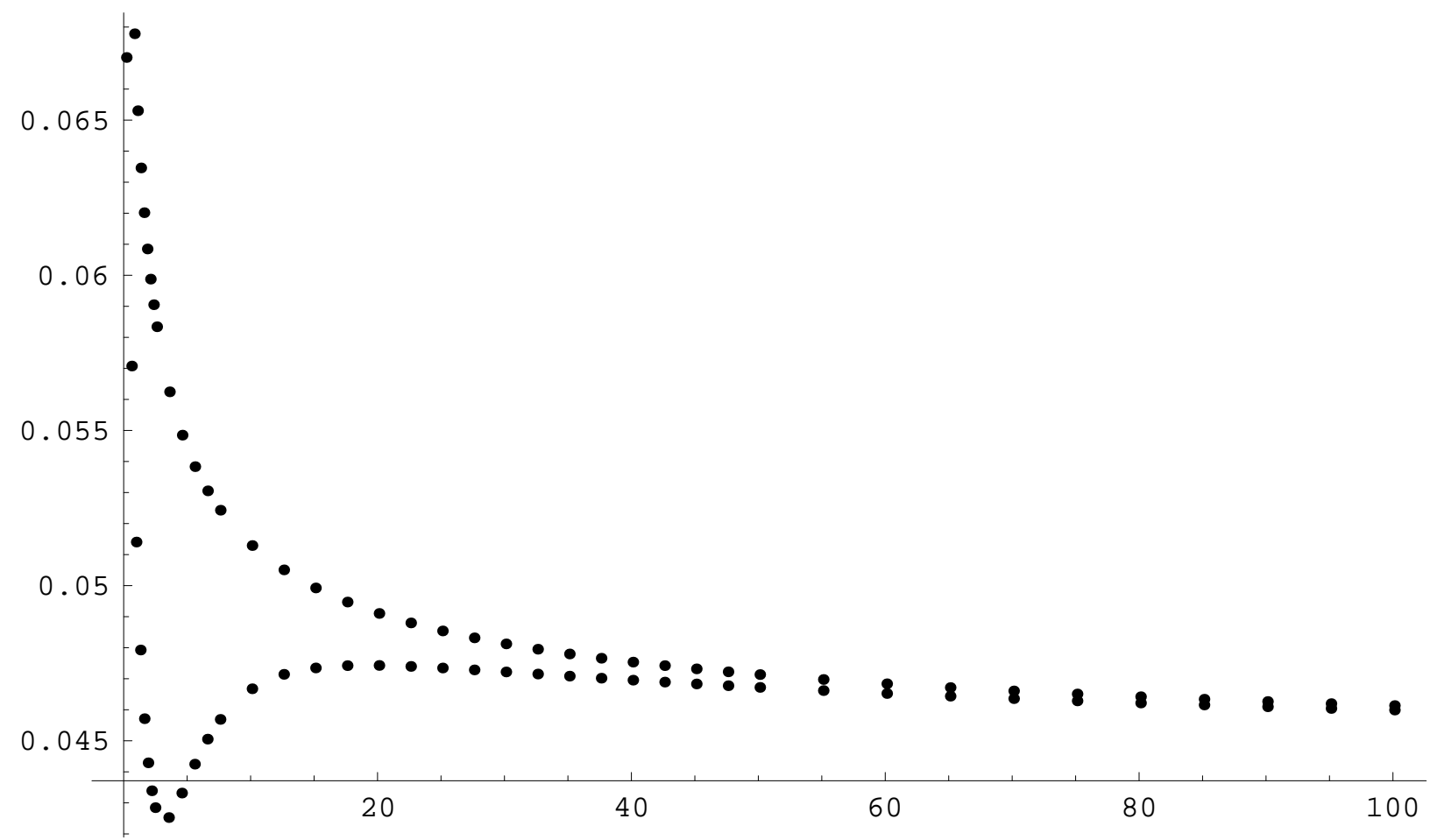

Figure 3: The real part of high overtones of $l=0, m=0.3$ and $m=3(\mathrm{M}=1)$ asymptotically approaches $\frac{\ln 3}{8 \pi}$

modes is infinite for each multi-pole number, the presence of any finite mass of the field will not induce disappearing of the whole spectrum, leaving a countable number of QN modes.

Note also, that the presence of the mass term does not change the asymptotic crucially and, in fact, QN frequencies found under boundary conditions (4) with the 6th order WKB approximation 10] do not differ from those found under the Leaver method where sub-dominant asymptotic term is taken into account, at least for not large values of $m$ (for more references on the usage of WKB method for finding QNMs see for instance [1].).

\section{High overtones}

First of all, let us propose some illustrative estimations for high-overtone behavior of the massive field. Starting from expansion of $\chi$ at high overtones in the following form

$$
\chi=\omega-\frac{m^{2}}{2 \omega}+O\left(\frac{1}{\omega^{3}}\right),
$$

and substituting (16) in (8), one can find

$$
\alpha_{n}(m)=\alpha_{n}(m=0) ; \quad \beta_{n}(m)=\beta_{n}(m=0)-16 M^{2} m^{2}+O\left(\frac{1}{\omega}\right) ; \quad \gamma_{n}(m)=\gamma_{n}(m=0)+O\left(\frac{1}{\omega}\right)
$$

We see that for high overtones $(M \operatorname{Im}(\omega) \gg 1)$, the behavior of coefficients is the same as in massless case with some shifted multi-pole number $l$. Reminding that asymptotical behavior of QNMs does not depend on the multi-pole number we can conclude that

$$
\omega(m, N)=\omega(m=0, N)+O\left(\frac{1}{\omega}\right)=\frac{N-1 / 2}{4 M} i+\frac{\ln 3}{8 \pi M}+O\left(N^{-1 / 2}\right) .
$$

If we do not pay attention to terms like $n / \omega$, this arguing illustrates why the asymptotical behavior of QNMs does not depend on the field mass. To prove this fact exactly, one should to reproduce all the 
steps in [15]. We omit them because they are almost exactly the same as in [15], but require additional discussions. Finally, we find the equation for high overtones that leads to the solution (17):

$$
\tan (2 M i \pi \omega) \tan ^{2}\left(M i \pi(\omega+\chi)^{2} / \chi\right)=\tan (2 M i \pi \omega) \tan ^{2}\left(2 M i \pi \omega+O\left(\frac{1}{\omega^{3}}\right)\right)= \pm i+O\left(\frac{1}{\sqrt{\omega}}\right) .
$$

Numerical calculations confirm our arguments. On figure 3 you can see that the difference between modes for $m=0.3$ and $m=3$ is small for high overtones. More accurately, we constructed fits for both curves and found that they tend to (17). (Fits and data for different $l$ are available from the second author upon request).

\section{Conclusion}

We have made a thorough investigation of the quasinormal spectrum for massive scalar field in a Schwarzschild black hole background. It is shown that the mass of the field has crucial influence on damping rate of the QNMs. In particular, the greater the mass of the field is, the less the damping rate. As a result, purely real modes appear which corresponds to non-damping oscillations, and, when the field mass is greater than some threshold values, the lower overtones disappear. This happens however with lower overtones only, while all the remaining higher overtones are still damping. Generally, we proved, that such arbitrary long living modes are forbidden, unless the effective potential is non-zero at least at some of the two boundaries (event horizon or spatial infinity). Note, that since we are working in a linear approximation and also since the QN modes do not form a complete set, the existence of arbitrary long living modes, apparently, does not mean we can really have a system without damping. We should expect that in the fully non-linear analysis the quasi-resonance modes may have non-vanishing damping rate.

In addition we proved that at asymptotically high overtones the real part of the QNMs goes to $\ln 3 /(8 \pi M)$ for any (finite) mass of the field. The numerical, analytical and WKB methods we used show excellent agreement in their range of validity. It is interesting for us to find out what will change in the above picture if considering massive fields of higher spin. The massive Dirac field represents apparently the most easy case.

\section{Acknowledgements}

The authors acknowledge Andrei Starinets for a most useful discussion. The work of R.K. was supported by FAPESP (Brazil).

\section{References}

[1] K.Kokkotas and B.Schmidt, "Quasi-normal modes of stars and black holes", Living.Reviews. Relativ. 2, 2 (1999). H-P. Nollert, Class.Quant.Grav. 16, 159 (1999).

[2] G.T.Horowitz and V.Hubeny, Phys. Rev. D62 024027 (2000); A.Starinets, Phys.Rev.D66:124013, 2002; K.H.C. Castello-Branco, R.A. Konoplya, A. Zhidenko, hep-th/0411055; S. Das, S. Shankaranarayanan, hep-th/0410209 R.A.Konoplya and A.Zhidenko, JHEP (06) 037 (2004) hep-th/0402080; V. Cardoso, R. Konoplya and J. P. S. Lemos, Phys.Rev. D 68044024 (2003); E. Berti, V. Cardoso and S. Yoshida, Phys.Rev.D69:124018,2004; R.A.Konoplya, Phys.Rev. D 66 084007 (2002); Phys.Rev.D70:047503,2004; Phys.Rev.D66:044009,2002; hep-th/0410057; G. Siopsis, Phys.Lett.B590:105-113,2004; M.R. Setare, Phys.Rev.D69:044016,2004; E. Berti V. Cardoso, K. D. Kokkotas and H. Onozawa, Phys.Rev.D68:124018, 2003;

[3] L. E. Simone, C. M. Will, Class.Quant.Grav.9 963-978, 1992

[4] R.A.Konoplya, Phys.Lett. B550 117, 2002 gr-qc/0210105.

[5] H.Koyama and A.Tomimatsu, Phys. Rev. D 63, 064032 (2001).

[6] H.Koyama and A.Tomimatsu, Phys. Rev. D 64, 044014 (2002). 
[7] L.Burko and G.Khanna, Phys. Rev. D 70, 044018 (2004).

[8] Li-Hui Xue, Bin Wang, Ru-Keng Su, Phys. Rev. D 66, 024032 (2002).

[9] B.R. Iyer, S. Iyer, C.V. Vishveshwara, Class.Quant.Grav.6 1627, (1989).

[10] S. Iyer and C. M. Will, Phys.Rev. D35 3621 (1987); R.A.Konoplya， Phys. Rev . D68 024018 (2003)

[11] R.A. Konoplya, Phys.Rev.D68:124017,2003, hep-th/0309030; R.A. Konoplya, Gen.Rel.Grav.34:329335,2002 gr-qc/0109096; Wei Zhou, Jian-Yang Zhu, Int.J.Mod.Phys.D13:1105-1118,2004 gr-qc/0309071.

[12] A. Ohashi and M. Sakagami, Class.Quant.Grav.21, 3973, (2004)

[13] E. M. Leaver, Proc. Roy. Soc. Lond. A402:285-298,1985

[14] Nollert H. -P. 1993 Phys. Rev. D 475253

[15] L. Motl, Adv.Theor.Math.Phys.6:1135-1162,2003

[16] Bachelot, A., and Motet-Bachelot, A., " Les resonancesdn trou noir de Schwarzschild", AA.Inst. Henri Poincare, 59, 3, (1993)

[17] A.Zhidenko, Class.Quant.Grav. 21273 (2004). 